\title{
ÉVOLUTION DES PLUIES DE CUMUL ÉLEVÉ ET RECRUDESCENCE DES CRUES DEPUIS 1951 DANS LE BASSIN DU NIGER MOYEN (SAHEL)
}

\author{
Luc DESCROIX ${ }^{12}$, Aïda DIONGUE NIANG ${ }^{3}$, Honoré DACOSTA ${ }^{4}$, \\ Gérémy PANTHOU $^{1}$, Guillaume QUANTIN ${ }^{1}$ et Arona DIEDHIOU ${ }^{1}$
}

\author{
${ }^{1}$ LTHE UMR 5564 - IRD / UJF-Grenoble 1 / CNRS / G-INP \\ BP 53, 38041 Grenoble cedex 9 - France \\ luc.descroix@ird.fr ; geremy.panthou@ete.inrs.ca ; guillaume.quantin@ujf-grenoble.fr ; arona.diedhiou@ird.fr \\ ${ }^{2}$ UMR 208 "Patrimoines Locaux" \\ Muséum National d'Histoire Naturelle - Département Hommes, Natures, Société \\ 57 rue Cuvier, CP 26 \\ 75231 Paris cedex 05 - France \\ ${ }^{3}$ Agence Nationale de l'Aviation Civile et de la Météorologie (ANACIM)/MET \\ Aéroport Léopold Sédar Senghor, BP 8257 Dakar-Yoff, Dakar - Sénégal \\ aida.diongue.niang@gmail.com \\ ${ }^{4}$ Département de Géographie, FLSH, Université Cheikh Anta Diop \\ BP 5005, Dakar - Sénégal \\ honore.dacosta@ucad.edu.sn
}

\begin{abstract}
Résumé
L'augmentation observée du ruissellement et des écoulements au Sahel depuis le début de la sécheresse constitue le "paradoxe hydrologique du Sahel". Il est attribué depuis sa mise en évidence dans les années 1980 au changement d'usage des sols, la combinaison de la mise en cultures, du raccourcissement des jachères et de la fragilisation des couverts végétaux par les pics de sécheresse ayant conduit à un encroûtement des sols. Ce dernier est à l'origine de l'augmentation des coefficients de ruissellement. Mais on a récemment observé une augmentation du nombre d'évènements pluviométriques de fort cumul précipité au Sahel. On se propose ici de déterminer si une telle évolution est perceptible sur le bassin moyen du fleuve Niger, et d'analyser, le cas échéant, si elle est susceptible de contribuer à l'accroissement des volumes écoulés observés. Cette région est la partie sahélienne du bassin, située à cheval sur le Burkina Faso, le Mali et le Niger; cette zone semi-aride recevant de 250 à $800 \mathrm{~mm}$ de pluie a été sévèrement touchée par la sécheresse après 1968. Sa végétation naturelle a été en grande partie détruite et/ou remplacée par des cultures. On utilise ici les données des précipitations journalières classées et par décennie pour analyser l'évolution des évènements de fort cumul pluviométrique depuis 1951. Le paradoxe du Sahel est renforcé depuis la ré-augmentation partielle des précipitations dans la fin des années 1990 par une augmentation plus forte des évènements journaliers à fort cumul pluviométrique.
\end{abstract}

Mots-clés : Sahel, crues, inondations, précipitations extrêmes, changement d'usage des sols.

\begin{abstract}
Evolution of rainy extreme events and rise in flooding in the Sahel

The increase in runoff and stream flows observed in the West African Sahel since the beginning of the Great Drought in 1968 constitutes the "Sahelian hydrological paradox". Since its highlighting in the 1980s, it has been shown to result from land use changes, a combination of land clearing for cropping, fallow shortening and the vegetation weakening by drought peaks during the 1970s and the 1980s, all factors leading to soil crusting. The latter is the main cause of the rise in runoff coefficients. However, an increase in the number of extreme rainfall events was recently observed. The main aim of this paper is to determine whether such an evolution is also noticeable in the Middle Niger River basin and to analyze whether, in this case, it could contribute to the observed increase in stream flows. We analysed data of the Sahelian part of the Niger Basin; this area overlapping on Burkina Faso, Mali and Niger, is a semi-arid zone, with annual rainfall ranging from 250 to 800 $\mathrm{mm}$. It suffered a severe drought after 1968, aggravating the natural vegetation decrease due to the increase in cropping areas. We analyze here daily precipitation data from 1951 to 2010 by decades to determine the evolution of high rainfall amount events. The "Sahelian Paradox" was recently reinforced by the increase in rainfall which results in a higher increase in the frequency of high rainfall amount events.
\end{abstract}

Keywords: Sahel, floods, inundations, extreme rainfall events, land use changes.

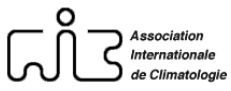




\section{Introduction}

L'accroissement des écoulements malgré le déficit des pluies observé depuis la fin des années 1960 est un processus qui s'observe dans l'essentiel du Sahel, d'où son nom de « paradoxe hydrologique du Sahel ». D'abord observé sur de petits bassins du Burkina Faso par Albergel (1987), il est confirmé par Mahé et Olivry (1999) dans plusieurs bassins d'Afrique de l'Ouest, puis par Amani et Nguetora (2002) et Olivry (2002) sur celui du Niger. C'est ensuite sur les affluents de rive droite de celui-ci (Mahé et al., 2003), puis sur le bassin du Nakambé (Mahé et al., 2005 et 2010) que la même hausse est observée. Gil Mahé et ses co-auteurs ont aussi mis en évidence ce comportement paradoxal à l'Ouest en Mauritanie (Mahé et Paturel, 2009) puis à l'Est (Mahé et al., 2011) dans la zone sahélienne du Nigéria. En zone pastorale nord-sahélienne, Gardelle et al. (2010) ont observé une forte croissance des mares, liée à l'augmentation du ruissellement, celle-ci étant due à la non-reconstitution de la végétation après la sécheresse dans les zones où le peu de sol a été emporté. Localement dans le degré carré de Niamey, le développement des mares en réponse à une augmentation du ruissellement (Bouzou et al., 2009) entraine une hausse du niveau des eaux souterraines ; en effet, les mares sont les principales zones de recharge de la nappe (Leduc et al., 2001).

On sait depuis les travaux d'Albergel (1987) qu'inversement, en zone soudanienne, les écoulements ont, plus logiquement, diminué avec la sécheresse, et ce, à l'échelle des bassins expérimentaux du Burkina. Ceci a été confirmé par Olivry (2002) puis Mahé (2009) et Amogu et al. (2010). Enfin, la crue du Niger Moyen en 2010 donne l'occasion à Descroix et al (2012) de souligner la relation entre l'encroûtement et l'accroissement du risque de crue et d'inondation en Afrique de l'Ouest. Elle montre comment l'urbanisation rapide y accentue le risque en décuplant la vulnérabilité des populations concentrées dans des zones pas toujours urbanisables.

\section{Le contexte}

Le fleuve Niger moyen (en aval du delta intérieur, figures 1 et 2) produit deux crues. La première, dite "crue locale" (ou crue rouge, elle doit sa couleur à sa forte charge solide venant de sols latéritiques), intervient en général pendant les mois d'août à septembre. Elle résulte des apports de plusieurs affluents dans cette partie du fleuve, dont principalement les affluents de la rive droite en amont de Niamey (Gorouol, Dargol, Sirba), qui prennent leur source au Burkina Faso. La seconde crue, beaucoup plus importante en volume et à pointe unique, est appelée "crue guinéenne" (ou crue noire). Elle résulte des eaux provenant de la partie amont du bassin du fleuve (Guinée et Mali) et se produit au cours de la période allant de novembre à mars.

$\mathrm{Si}$, comme on l'a vu précédemment, les changements d'usage des sols semblent faire consensus comme principale cause de l'augmentation des ruissellements, des travaux récents ont pu montrer le rôle de l'ensablement du lit du Niger comme facteur aggravant des crues, les transformant en inondations, ainsi que celui de l'accroissement de la taille du bassin du fleuve, dû aux ruptures d'endoréisme (Amogu et al., 2010 ; Mamadou, 2012 ; Descroix et al., 2012 ; Sighomnou et al., 2013).

Enfin, Panthou (2013) a analysé les pluies journalières dans une fenêtre allant de $10^{\circ} \mathrm{W}$ à $5^{\circ} \mathrm{E}$ et de $10^{\circ} \mathrm{N}$ à $15^{\circ} \mathrm{N}$, pour étudier l'évolution des précipitations extrêmes. Il a montré un accroissement récent du nombre d'évènements pluviométriques "extrêmes » (en fait, des évènements de fort cumul pluviométrique) depuis le milieu des années 2001-2010. Par ailleurs, Panthou et al. (2013) ont mis en évidence la baisse du nombre de ces épisodes lors de la période de déficit pluviométrique, et sa remontée récente. 
L'objectif de cet article est de déterminer si une telle croissance des évènements de fort cumul pluviométrique s'observe sur le bassin du Niger Moyen, et si elle peut être une cause de l'accroissement des débits et de la recrudescence récente des inondations dans cette région. Il convient en particulier de déterminer si les pluies sont à présent plus nombreuses ou intenses que celles des décennies « humides » (1951-1968).

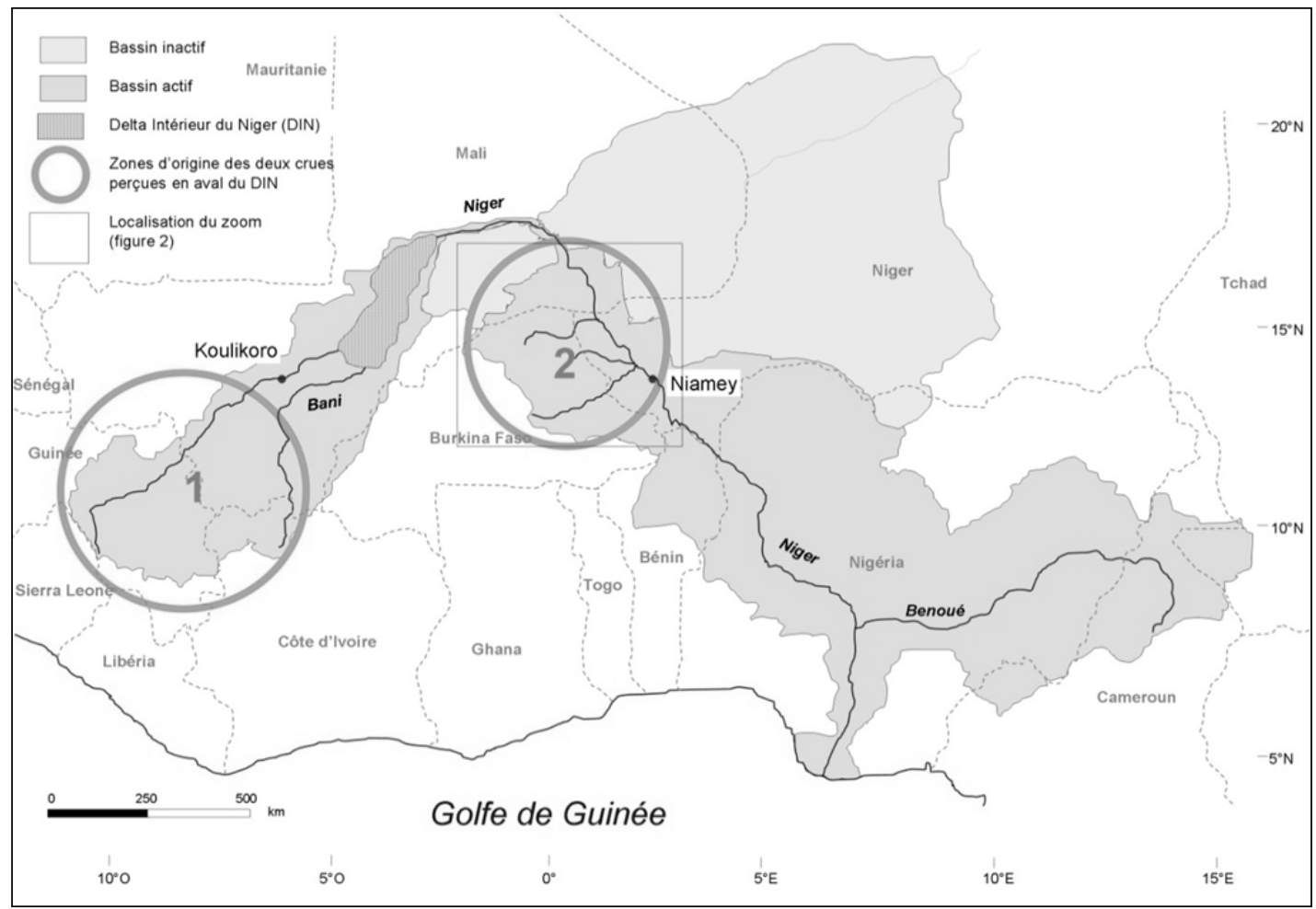

Figure 1 : Le bassin du Niger et ses deux principales zones d'apports d'eau; $1:$ les montagnes de Guinée ; $2:$ le bouclier granitique du Liptako-Gourma. The Niger River basin and its two main providing water areas; 1: Guinean mountains; 2: the Liptako-Gourma granitic shield.

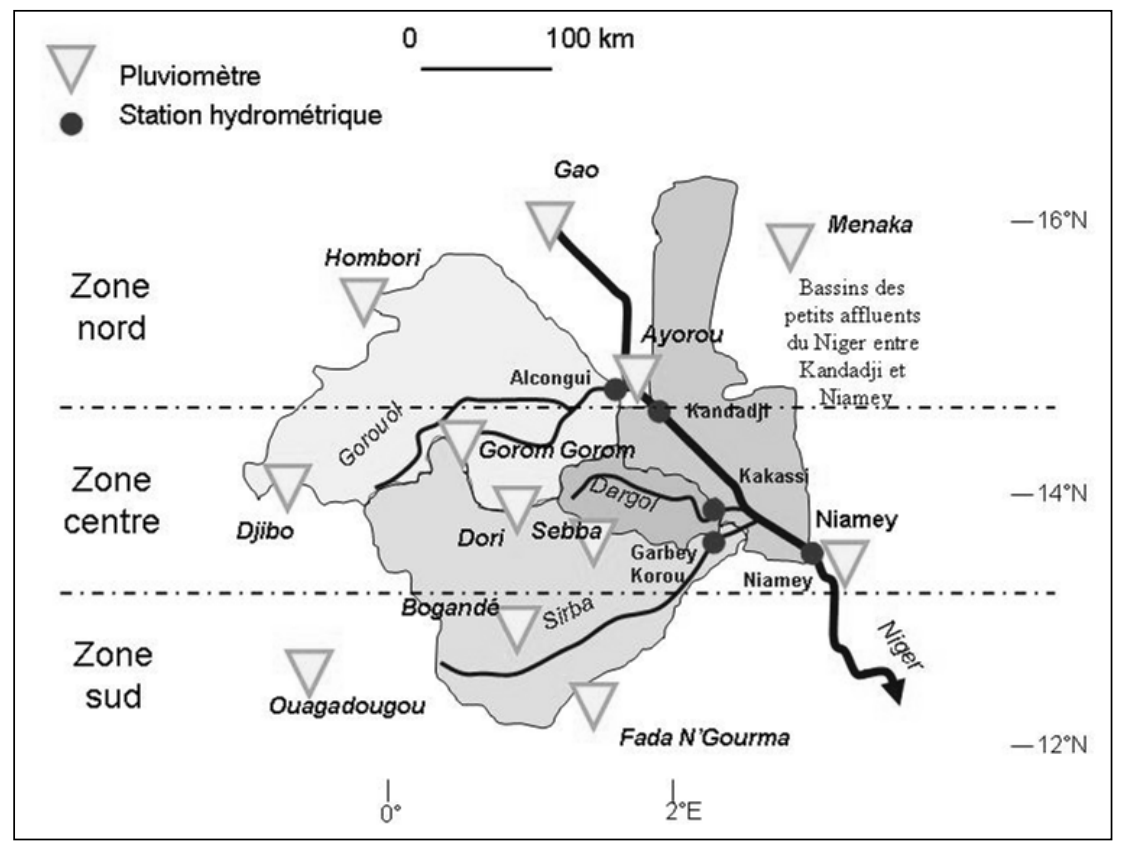

Figure 2 : Le bassin moyen du fleuve Niger : les stations hydrométriques et les pluviomètres dont les données sont utilisées ici. The middle Niger River basin: stream gauges and rain gauges whose data are used in this study. 


\section{Le réseau de mesures}

On s'intéresse ici aux pluies de fort cumul (plus de 20, 30, 40, 50 et $60 \mathrm{~mm}$ ) observées dans le bassin moyen du fleuve Niger; il s'agit de la partie exoréique du bassin située en aval du delta intérieur du Niger et en amont de Niamey. Les débits sont mesurés, pour ce bassin moyen, principalement à Kandadji et à Niamey sur le fleuve Niger, et à trois stations contrôlant les trois affluents principaux, provenant tous trois de la rive droite, c'est-à-dire du bouclier granitique du Liptako-Gourma (figure 2). En effet, en rive gauche, affleure l'extrémité ouest du vaste bassin sédimentaire des Iullemeden, caractérisé par une dégradation hydrologique et l'endoréisme.

Nous avons utilisé par ailleurs les données de la base AMMA-CATCH pour établir sur le bassin du Niger Moyen un indice pluviométrique annuel qui représente pour chaque année l'écart à la moyenne de la série de la lame précipitée. Les pluies sont mesurées par une cinquantaine de pluviomètres, dont les deux tiers en territoire burkinabé; mais nous n'utilisons ici que les données de douze stations dont les enregistrements sont de qualité et ininterrompus depuis 1950 (figure 2). Ces séries sont complètes sur la période de 60 ans mais a contrario, le réseau est lâche pour une aire de $100000 \mathrm{~km}^{2}$; la densité est d'un poste pour $8000 \mathrm{~km}^{2}$ environ, soit un peu plus d'un par degré carré.

Les pluies journalières ont été classées par année et par station de manière à établir le nombre d'évènements ayant atteint une classe donnée de cumul pluviométrique, ainsi que, pour chaque classe et chaque station, le cumul pluviométrique fourni par les évènements considérés.

Les travaux de Panthou (2013) ont porté sur une grande fenêtre (voir ci-dessus); nous nous intéresserons ici à une zone bien plus petite (environ $4^{\circ}$ du nord au sud et de l'ouest à l'est) car notre but est de mettre en relation cette évolution des épisodes de fort cumul pluviométrique avec celle des débits sur trois affluents de rive droite du fleuve Niger. Pour compenser le plus faible nombre des stations, nous avons réalisé cette étude par décade de manière à obtenir un échantillon d'évènements statistiquement satisfaisant. Par ailleurs, nous apportons une analyse intra-saisonnière de cette évolution, réalisée ici par mois et non plus seulement par saison.

\section{Ce que l'on observe}

\subsection{Une augmentation des écoulements}

Depuis quelques décennies, certains affluents du fleuve Niger semblent enregistrer une activité particulière, avec des ruptures d'endoréisme conduisant à un accroissement de la surface de l'impluvium du bassin du Niger. Traditionnellement, le débit du fleuve Niger diminuait jusqu'à Niamey. Amogu et al. (2010) ont remarqué que depuis la fin des années 1990, les débits ré-augmentaient dès en amont de Niamey, les débits à cette station dépassant désormais ceux enregistrés à Kandadji (figure 2). Suite aux observations d'Albergel (1987), les nombreux travaux de Mahé et al. (cités auparavant), ainsi que Amogu et al. (2010) ont montré que l'augmentation des débits était le cas sur l'ensemble du Sahel; mais qu'elle ne concernait effectivement que le Sahel et, pour les dernières années, le nord de la zone soudanienne.

Toujours est-il que les trois principaux affluents sahéliens du fleuve Niger (le Gorouol, le Dargol et la Sirba, du nord au sud) sont tous de rive droite; ils proviennent du bouclier granitique du Liptako-Gourma, qui représente un modeste château d'eau régional mais dont la production d'écoulement est en augmentation notoire. Tout comme le Nakambé, qui vient 
aussi de ce bouclier granitique et est également sahélien (Mahé et al., 2005), ces trois cours d'eau ont vu leur débit et leur coefficient d'écoulement augmenter très fortement au cours des dernières décennies; depuis le début de la sécheresse que connaît l'Afrique de l'Ouest, les débits ont été multipliés par 2,5 à 3,5 (figure $3 \mathrm{a}$, avec les lames écoulées des trois sousbassins), les coefficients d'écoulement par 3 ou 4 (figure 3b).

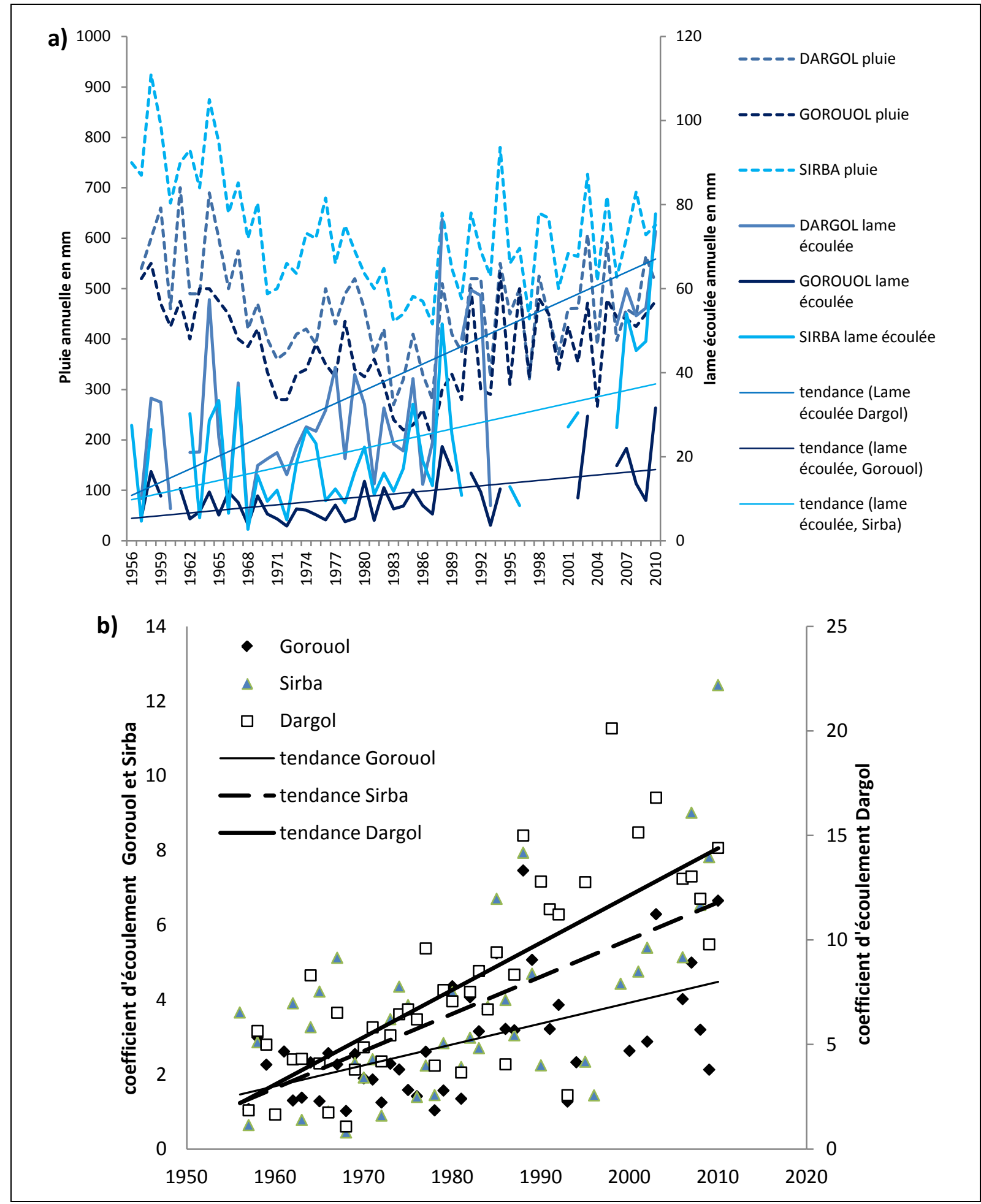

Figure 3 : a) Evolution des pluies et des lames écoulées sur les bassins des affluents sahéliens du Niger depuis 1956 ; b) Augmentation des coefficients d'écoulement des mêmes basins. a) Increase in rainfall and runoff depth; b) Increase in runoff coefficients of the main Sahelian Niger River tributaries since the beginning of the Drought. 
Les figures 3 et 4 montrent bien que cette augmentation a commencé au début de la sécheresse (fin des années 1960) ; il est probable que la forte mortalité des ligneux observée alors a causé une baisse de la capacité de rétention en eau des bassins, exagérée par la surexploitation du milieu.

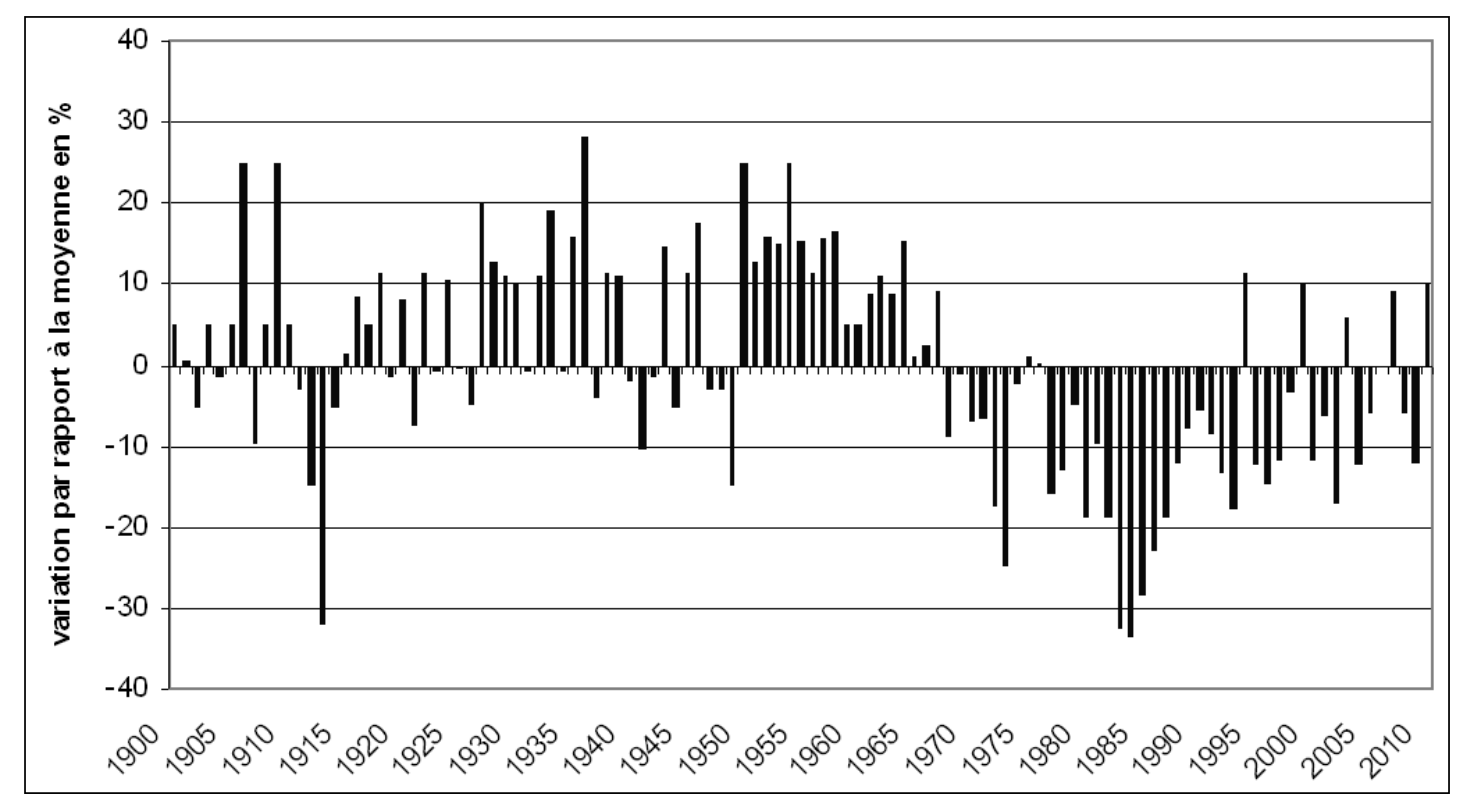

Figure 4 : L'évolution de l'indice des pluies depuis 1900 sur le bassin moyen du fleuve Niger (en \% de variation autour de la moyenne). Evolution of the rainfall index since 1900 over the Middle Niger River basin (in \% of variation / mean 1900-2010).

En effet, les rendements céréaliers étant très faibles sur les sols sableux du Sahel (moins de $500 \mathrm{~kg} / \mathrm{ha} / \mathrm{an}$ ), la forte croissance démographique conduit les paysans à diminuer les temps de jachère et à accélérer les rotations ; on considère que cette accélération d'un système cultural adapté à une faible densité de population est la cause principale de la dégradation des sols et de leur perte de fertilité et de capacité de rétention en eau. La mise en culture laisse le sol à nu durant la saison sèche, le soumettant à l'action de l'érosion éolienne, d'une part, et d'autre part, le raccourcissement des jachères reste la cause principale de la dégradation des sols cultivés (Van de Watt et Valentin, 1992 ; Ambouta et al., 1996).

\subsection{Une augmentation des évènements extrêmes}

Panthou et al. (2013) ont constaté, à l'échelle d'une grande partie centrale du Sahel, une augmentation récente du nombre d'évènements pluvieux qu'ils nomment « extrêmes » (en fait de fort cumul pluviométrique). Les figures $5 \mathrm{a}$ et $5 \mathrm{~b}$ montrent qu'il en est de même pour le bassin du Niger Moyen : le nombre d'évènements pluvieux des classes de cumul supérieures à $20,30,40,50$ et $60 \mathrm{~mm}$ augmente, ainsi que, de manière légèrement plus prononcée, le cumul pluviométrique concernant ces évènements-là.

Nous avons spatialisé l'information en fonction de polygones de Thiessen. La figure 6a montre que le bassin du Niger moyen a connu la même évolution que celle signalée par Le Barbé et al (2002) : la pluviométrie y a baissé fortement durant les décennies 1970 et 1980, en relation avec une diminution du nombre de jours de pluie. De fait, si la hauteur moyenne de pluie tombée par évènement a globalement peu évolué, elle a pratiquement rattrapé son niveau des décennies humides 1951-1970 : elle était alors de $13 \mathrm{~mm} \cdot \mathrm{j}^{-1}$ puis a diminué à 11,5 mm.j ${ }^{-1}$ entre 1971 et 1990 ; elle a atteint à nouveau $13 \mathrm{~mm} \cdot \mathrm{j}^{-1}$ dans la dernière décennie 20012010 . 


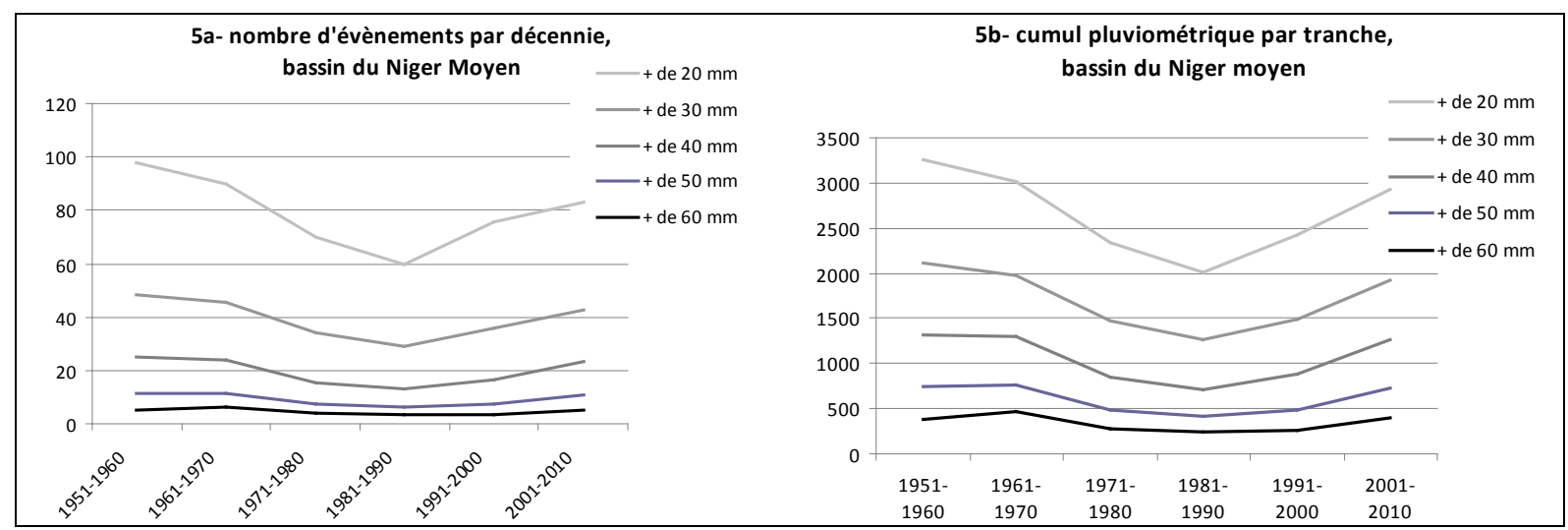

Figure 5 : L'évolution des précipitations par seuil de cumul tombé, et par décennie, depuis $1950:$ a) nombre d'évènements pluvieux; b) cumul de pluie tombée lors de ces évènements, par classe. Evolution of rainfall events per class of rainfall amount, per decade since 1950: a) number of rainy events; b) cumulative rainfall amount of these events, per class.

On peut aussi constater que les pluies de plus forts cumuls (plus de 40 et $50 \mathrm{~mm}$ respectivement) ont atteint durant la décennie 2001-2010 les plus hauts niveaux qu'elles avaient dans les années 1951-1970, tant en nombre d'évènements qu'en cumul des précipitations correspondant à ces évènements (figure 6b). Cependant, les niveaux des années 1951-1970 n'ont pour l'heure pas été dépassés.
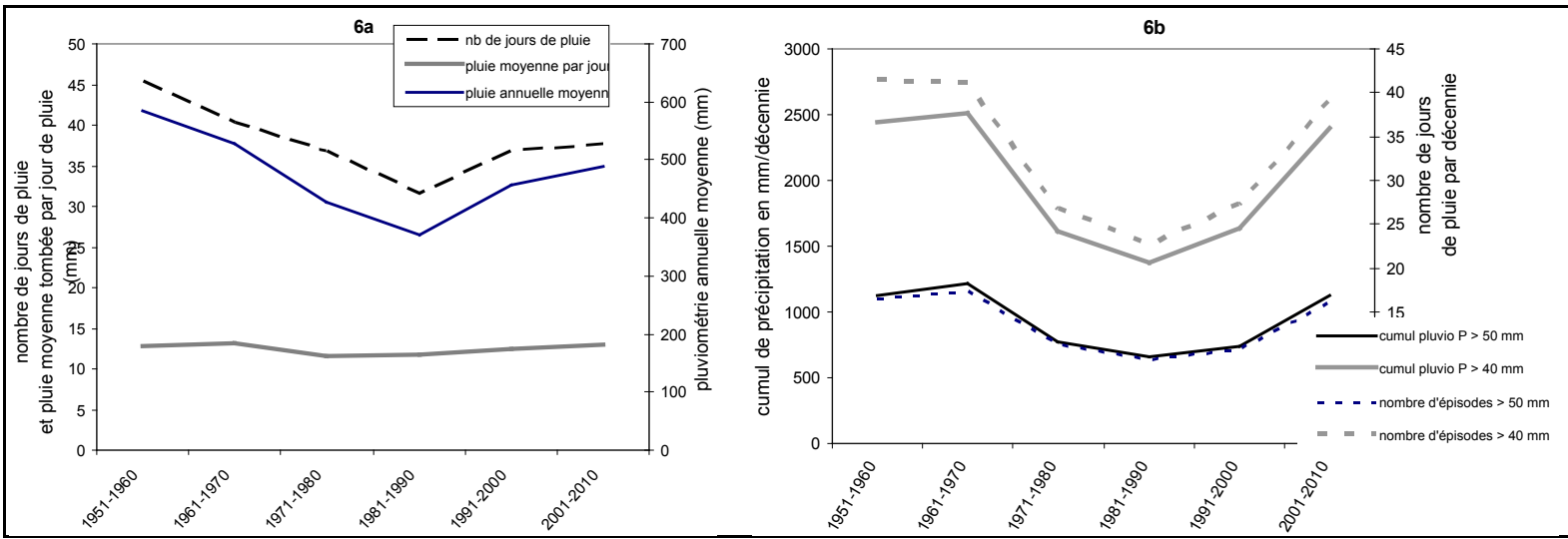

Figure 6 : a) L'évolution des précipitations, du nombre de jours de pluie et du montant de la pluie tombée, moyens, par décennie ; b) Evolution du nombre de jours de pluie de plus de 40 et $50 \mathrm{~mm}$ ainsi que du cumul pluviométrique correspondant. a) Evolution of the mean annual rainfall, the annual number of rainy events, the yearly average rainfall amount per event, mean per decade. b) Evolution of the number of rainy events $>40$ and $50 \mathrm{~mm}$ and corresponding rainfall amount.

\subsection{Une augmentation des évènements de fort cumul pluviométrique en début de saison}

La crue rouge se produit de plus en plus tôt, avec une quarantaine de jours d'avance actuellement par rapport à ce qu'on observait il y a une cinquantaine d'années; ceci est attribué à la disparition d'une grande partie de la végétation (Amani et Nguetora, 2002). La répartition des pluies extrêmes pourrait-elle aussi contribuer à expliquer cette précocité de la crue ? La figure 7 montre que le nombre d'évènements extrêmes remonte pour tous les mois sur la dernière décennie ou parfois, sur les deux dernières décennies. Mais les figures $7 \mathrm{a}$ et $7 \mathrm{~b}$ montrent qu'en début de saison des pluies, cette remontée des évènements extrêmes est plus prononcée, au point de rejoindre leur nombre observé durant les décennies humides. De plus, le cumul de pluie tombée pour ces évènements a même dépassé celui des années 1950 et 1960, cette croissance est d'autant plus forte que le seuil de précipitation considéré est élevé (figure 7b). Cette tendance se remarque encore, bien qu'atténuée, en juillet (figures 7c et 7d) ; 
par contre, août et septembre montrent une augmentation plus faible du nombre et du cumul des précipitations extrêmes, qui ne leur permet pas d'atteindre leur niveau des décennies humides 1951-1970 (figures 7e à 7h), en cohérence avec la baisse des précipitations d'août déjà montrée par Nicholson (2005).

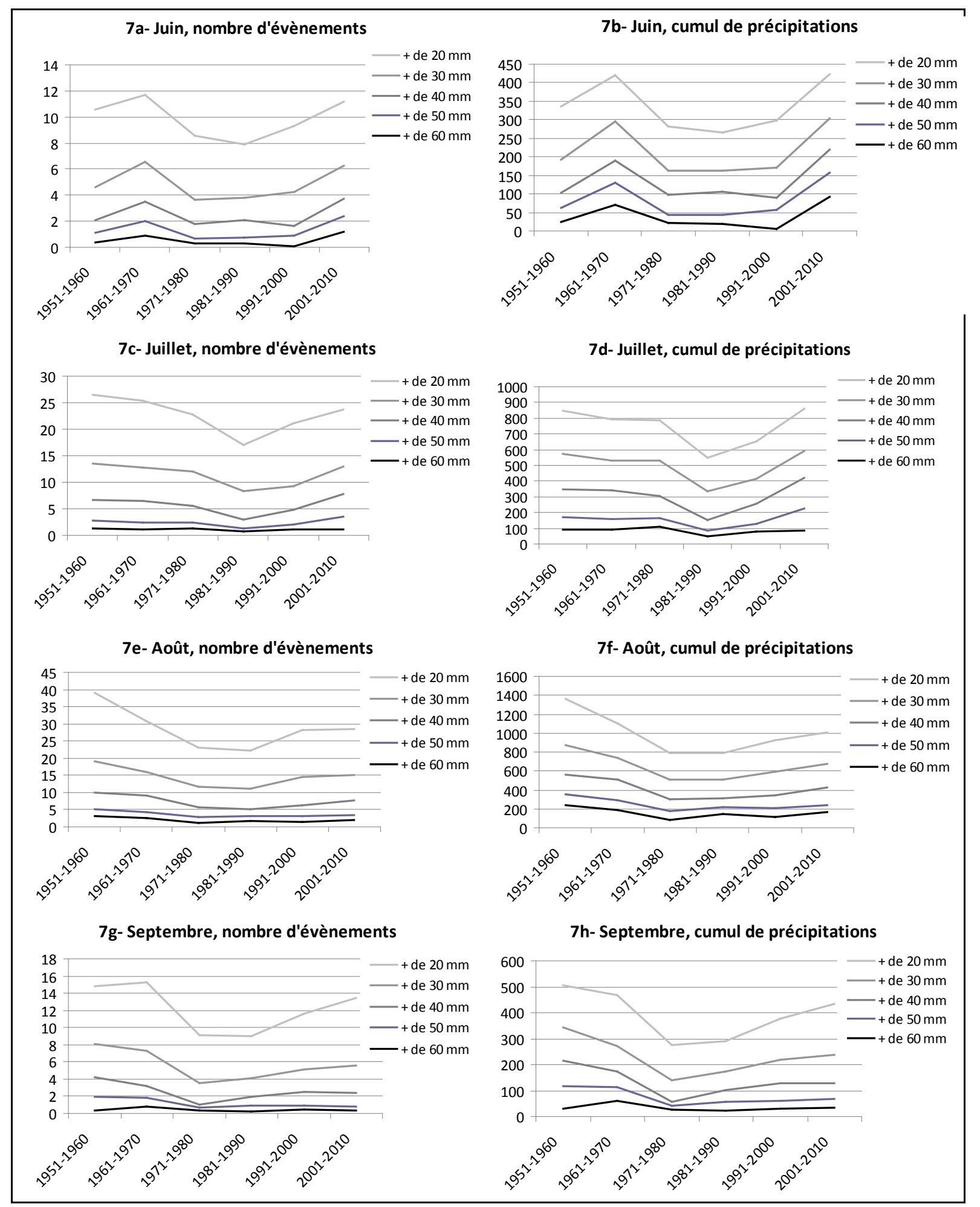

Figure 7 : L'évolution du nombre de jours de pluie et du cumul de précipitations par tranche de cumul et par décennie, de 1951 à 2010. Evolution of the number of rainfall events and the total rainfall amount per class of amount and per decade, from 1951 to 2010. 


\subsection{Des nuances régionales}

La figure 8 montre l'évolution du nombre d'évènements extrêmes et du cumul concerné dans les sous-régions nord (figures $8 \mathrm{a}$ et $8 \mathrm{~b}$ ), centre (figures 8c et 8d) et sud (figures $8 \mathrm{e}$ et $8 \mathrm{f}$ ) ; voir la délimitation des trois régions sur la figure 2. On remarque que la baisse puis la remontée du nombre d'évènements et leur cumul sont plus marquées au nord et au centre que dans la sous-région sud. Par ailleurs, si la baisse est très forte dans les décennies 1970-1980 dans les deux régions nord et centre, la remontée dans la zone nord est moins nette et semble marquer une pause durant la décennie 2000 .

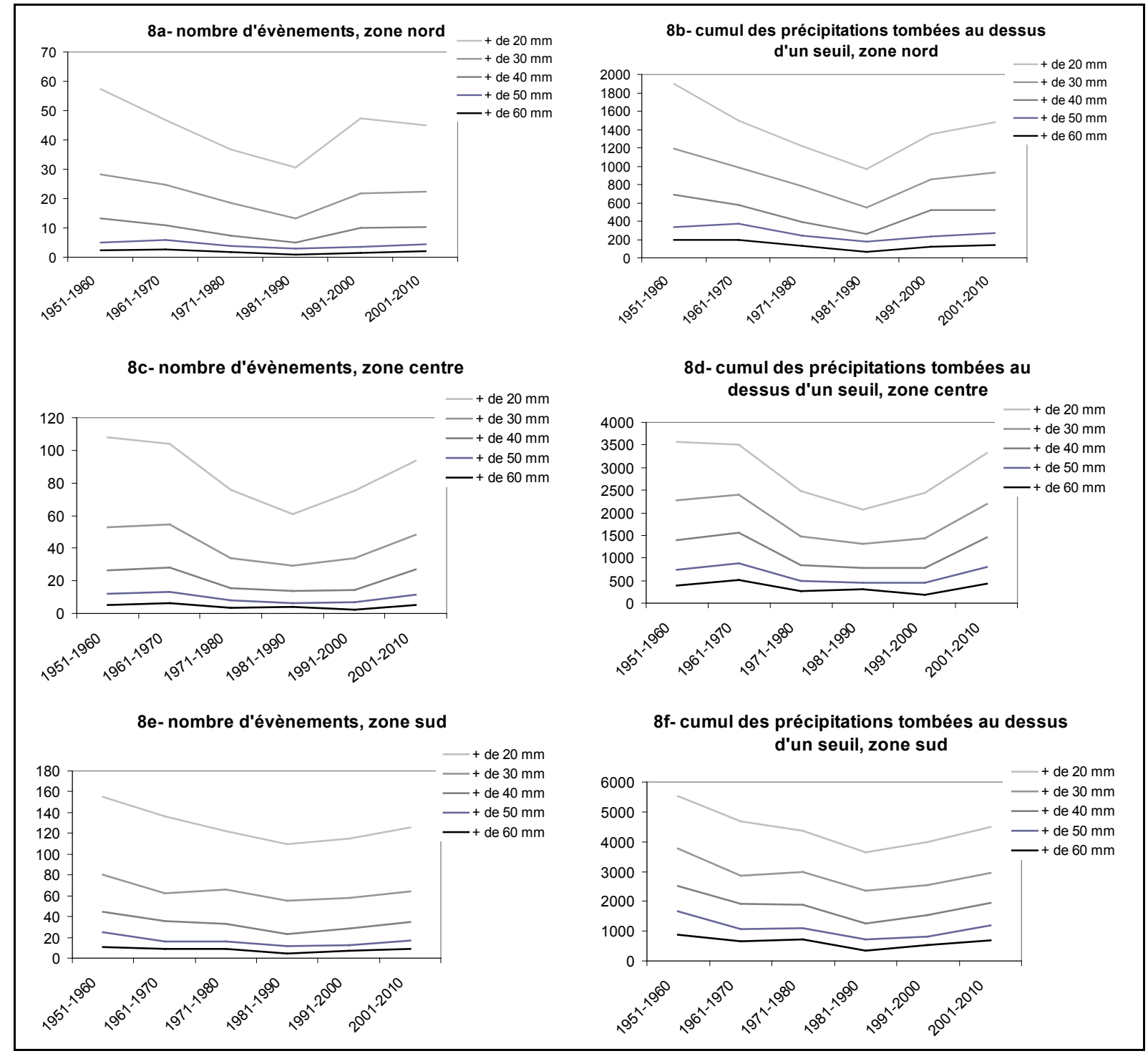

Figure 8: L'évolution du nombre de jours de pluie (par seuil de hauteur de pluie) et du cumul des précipitations concernées, par sous-région : Nord (a et b), Centre (c et d), Sud (e et f). Evolution of the annual number of rainy days (per threshold of rainfall amount) and the rainfall cumulative amount, per sub-region: Northern ( $a$ and $b$ ), Central (c and d), Southern (e and f).

Il faut noter toutefois que la petite taille de l'échantillon (en particulier pour les pluies de plus de 50 ou $60 \mathrm{~mm}$ par jour) et la grande variabilité spatiale de la pluie, empêchent de généraliser et d'étendre géographiquement cette constatation.

Le tableau 1 montre l'occurrence des évènements de fort cumul à trois stations situées dans la zone nord (Gao), la zone centre (Niamey) et la zone sud (Fada N'Gourma). L'augmentation 
de l'occurrence vers le sud est très forte, en relation avec l'augmentation des précipitations annuelles moyennes et le nombre de jours de pluie par an.

\begin{tabular}{|ccccccc|}
\hline $\begin{array}{c}\text { Nombre } \\
\text { d'évènements par an }\end{array}$ & latitude & $\mathbf{+ 2 0} \mathbf{~ m m}$ & $\mathbf{+ 4 0} \mathbf{~ m m}$ & $\mathbf{+ 6 0} \mathbf{~ m m}$ & $\mathbf{+ 1 0 0} \mathbf{~ m m}$ & $\begin{array}{c}\text { Pluie journalière } \\
\text { max 1951-2010 }\end{array}$ \\
\hline Gao & $16^{\circ} \mathrm{N}$ & 2,7 & 0,5 & 0,1 & 0,03 & 119 \\
\hline Niamey & $13.5^{\circ} \mathrm{N}$ & 9 & 2,1 & 0,5 & 0,06 & 173 \\
\hline Fada & $12^{\circ} \mathrm{N}$ & 14 & 4,1 & 1 & 0,09 & 135,5 \\
\hline
\end{tabular}

Tableau 1 : Fréquence en nombre d'évènements par an, des épisodes pluvieux. Frequency of rainy episodes (in number of events a year).

\section{Discussion}

Une augmentation du cumul annuel total des pluies est observée depuis le milieu des années 1990, au moins sur le centre et l'est du Sahel (Ali et Lebel, 2009). Elle ne permet pas de rattraper les niveaux des décennies « humides », sauf en début de saison, et en particulier dans le nord, alors que les débits observés, eux, sont bien plus élevés que durant ces décennies-là.

En parallèle, dans le bassin du Niger Moyen, il y a une augmentation du nombre des évènements pluviométriques extrêmes, comme cela a été observé récemment (Panthou, 2013). Celle-ci est récente, marquée uniquement dans la fin de la décennie 2000. On ne peut donc pas vraiment parler de tendance ou d'évolution, mais cela pourrait bien être le début d'un changement; et elle ne peut s'observer sur nos données, qui s'arrêtent en 2010 et qui sont groupées par décennie pour des raisons statistiques.

Cette tendance avait déjà été pressentie localement au Niger dès 2006 par Abou Amani (comm. pers.). De plus, dans les dernières années, certains évènements ont marqué la sousrégion ouest-africaine, comme les graves inondations de 2007 du Nord Togo, Nord Ghana et sud du Burkina Faso (Tschakert et al., 2010). Deux ans après, le $1^{\mathrm{er}}$ septembre 2009, des précipitations exceptionnelles sont observées sur la ville de Ouagadougou (plus de $250 \mathrm{~mm}$ en quelques heures), qui auraient un temps de retour de 10000 ans (Karambiri, 2009), et sur la ville d'Agadès (Nord Niger), envahie par les eaux du Kori Agadès dont la digue a rompu. En fin juillet, puis fin août 2010, par deux fois, le fleuve Niger dépasse son niveau jamais atteint pour une crue rouge à Niamey; pour cet évènement, on a pu montrer que ce sont des pluies moyennes qui l'ont provoqué, mais tombant sur des sols de plus en plus encroûtés et ayant perdu leur capacité de rétention en eau (Descroix et al., 2012). En 2012, le mois d'août voit encore deux événements hydrologiques notoires se succéder; le 21 août, le fleuve Niger charrie près de $2500 \mathrm{~m}^{3} / \mathrm{s}$ à Niamey, atteignant son plus haut niveau jamais mesuré depuis 1929. Les dégâts sont considérables et 80 personnes perdent la vie dans cette grave inondation. Le 26 août, la ville de Dakar, plateau basaltique dominant l'océan d'une vingtaine de mètres, voit une grosse partie des bas-fonds de sa moitié Nord submergés par de l'eau d'une averse de $161 \mathrm{~mm}$ tombée en moins de deux heures, dont $144 \mathrm{~mm}$ tombés en 51 minutes ; ce n'est pas le record de cumul pluvieux enregistré (mais la deuxième hauteur maximale journalière jamais observée), par contre, l'intensité dépasse tous les extrêmes enregistrés jusque là (Dacosta, 2012).

Il semble qu'il y ait une augmentation des épisodes de fort cumul pluviométrique sans retour à une pluviométrie «normale» ou du moins, tout à fait comparable à celle des décennies «humides » (1950-1970). Dans les villes ou les régions dont les surfaces ont perdu leur capacité d'infiltration et sont en partie encroûtées, cette évolution pourrait être favorable à une augmentation des fortes crues dans les prochaines années. 
L'augmentation des évènements de fort cumul pluviométrique en début de saison (voir chapitre 3.3.) - qu'il faudra confirmer avec plus de stations et plus de temps, car le nombre d'évènements concernés reste statistiquement faible, pourrait accentuer le processus d'avancée de la crue dans le temps. Amani et Nguetora (2002) avaient déjà noté que la crue de la Sirba avait tendance à se produire de plus en plus tôt, comme témoignage de la baisse de la capacité de rétention en eau des bassins. Descroix et al. (2012) ont montré que la crue du Niger intervenait à présent 40 jours plus tôt que durant les «décennies humides". L'augmentation du nombre d'évènements de cumul élevé noté en début de saison pourrait bien expliquer l'accélération de ce mouvement noté durant la dernière décennie.

Cependant, pour l'heure, ni le nombre des épisodes de pluie de fort cumul, ni les cumuls de pluie correspondants n'ont dépassé les niveaux des années « humides » 1951-1970, sauf pour les mois de mai-juin, peu abondants en pluies. La très forte hausse des débits des affluents de droite du Niger est donc plutôt liée à la baisse de la capacité de rétention en eau des bassins et à l'augmentation conséquente des coefficients d'écoulement.

\section{Conclusion}

On sait depuis plus de 20 ans que les débits des cours d'eau sahéliens avaient augmenté à partir du début de la sécheresse qui touche l'Afrique de l'Ouest depuis 1968. Les scientifiques ont vite fait le lien avec la disparition rapide de la couverture végétale naturelle, principalement due au défrichement nécessaire pour accroître les productions vivrières, mais aussi liée à la surmortalité des ligneux causée par la succession d'années sèches. L'explication de ce "paradoxe hydrologique » semble faire de plus en plus consensus. L'encroûtement des sols dû à leur «fatigue » elle-même liée à la surexploitation et au raccourcissement des jachères, seul moyen de reconstitution de la structure et de la fertilité des sols sableux et pauvres en matière organique du Sahel, semble bien être à l'origine d'un doublement, voire d'un triplement, des débits des principaux affluents sahéliens du Niger.

Cependant, depuis la fin des années 2000, la recrudescence des crues et des inondations conduit à se demander si d'autres facteurs ne viendraient pas se greffer sur ce «bruit de fond " de la baisse de la capacité de rétention en eau des sols. En effet, l'expansion de l'exoréisme est une piste intéressante pour expliquer l'augmentation des volumes écoulés ; enfin, l'ensablement est à retenir comme cause possible des inondations.

Il semble par ailleurs que de plus en plus d'évènements de cumul pluviométrique élevé se produisent et pourraient expliquer la hausse des coefficients d'écoulement et des débits. On observe aussi une arrivée plus précoce de la crue, qui pourrait bien être liée à la conjonction de l'encroûtement des sols (cause anthropique) et de l'augmentation de l'occurrence des évènements pluviométriques extrêmes, surtout en début de saison des pluies (mai et juin). Toutefois, si l'augmentation du risque à cause de la hausse de la vulnérabilité des populations, tout comme l'accroissement des coefficients de ruissellement, font l'objet d'un consensus, l'augmentation des évènements pluvieux de cumul élevé, réellement observée, est récente et mérite d'être analysée en détail pour en déterminer la contribution exacte dans l'augmentation du nombre des inondations.

Remerciements : Ce travail a été rendu possible grâce à l'appui du projet « Niger-Hycos » de l'Autorité du Bassin du Niger (ABN) à Niamey (http://nigerhycos.abn.ne/useranon/htm/listStationByGroup.php), des Services hydrologiques Nationaux du Mali, du Burkina Faso et du Niger qui nous ont fourni les données de débit du Niger. Nos remerciements vont également à nos collègues des Directions Nationales des Météorologies de ces mêmes pays, les animateurs du réseau FRIEND ainsi que ceux de AMMA-Mali et

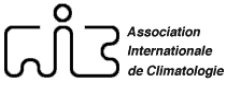


AMMA-Niger pour les données météorologiques. Les données du SIEREM nous ont été fournies par A. l'Aour-Crès, N. Rouché et C. Dieulin (IRD-HSM). Outre l'AFD qui finance la collecte des données hydrométriques de Niger-HYCOS, ce travail a été financé par les programmes ANR ECLIS et ANR-10-CEPL-005 ESCAPE, ainsi que par la JEAI (Jeune Equipe Associée à l'IRD) "Savane Parc à Long Terme », animée par le département de géographie de l'Université de Niamey.

\section{Bibliographie}

ALBERGEL J. 1987 : Sécheresse, désertification et ressources en eau de surface : application aux petits bassins du Burkina Faso. In The Influence of Climate Change and Climatic Variability on the Hydrologic Regime and Water Resources; IAHS publication $\mathrm{n}^{\circ} 168$, Wallingford, UK, 355-365.

ALI A., LEBEL T., 2009 : The Sahelian standardized rainfall index revisited. Int. J. Climatol. 29, 1705-1714. doi: 10.1002/joc.1832.

AMANI A., NGUETORA M., 2002 : Evidence d'une modification du régime hydrologique du fleuve Niger à Niamey. In FRIEND 2002 Regional Hydrology: Bridging the Gap between Research and Practice, Proceedings of the Friend Conference, Cape Town, South Africa, 1822 March, 2002; Van Lannen, H., Demuth, S., Eds.; IAHS publication $n^{\circ} 274$, Wallingford, UK, 449-456. http://iahs.info/redbooks/a274/iahs_274_449.pdf.

AMBOUTA J.-M.-K., VALENTIN C. et LAVERDERE M., 1996 : Jachères et croûtes d'érosion au Sahel. Sécheresse, 7, 269-75.

AMOGU O., DESCROIX L., SOULEY YERO K.S., LE BRETON E., MAMADOU I., ALI A., VISCHEL T., BADER J.-C., MOUSSA I.B., GAUTIER E., BOUBKRAOUI S., BELLEUDY P., 2010 : Increasing River Flows in the Sahel? Water, 2(2), 170-199.

BOUZOU MOUSSA I, FARAN MAIGA O, AMBOUTA J.-M.-K., SARR B., DESCROIX L., ADAMOU M., 2009 : Les conséquences géomorphologiques de l'occupation des sols et des changements climatiques dans un bassin versant rural sahélien. Sécheresse, 20(1), 1-8.

DACOSTA H., 2012: Les caractéristiques de la pluie du 26 août 2012 à Dakar. Note technique UCAD-DMN.

DESCROIX L., GENTHON P., AMOGU O., RAJOT J-L., SIGHOMNOU D., VAUCLIN M., 2012 : Change in Sahelian Rivers hydrograph: The case of recent red floods of the Niger River in the Niamey region. Global Planetary Change, 98-99, 18-30.

GARDELLE J., HIERNAUX P., KERGOAT L. \& GRIPPA M., 2010 : Less rain, more water in ponds: a remote sensing study of the dynamics of surface waters from 1950 to present in pastoral Sahel (Gourma region, Mali). Hydro. Earth Syst. Sci., 14, 309-324. doi:10.5194/hess14-309-2010.

KARAMBIRI H. 2009 : Brève analyse fréquentielle de la pluie du $1^{\text {er }}$ septembre 2009 à Ouagadougou (Burkina Faso). Note technique 2iE, 4 p.

LE BARBE L., LEBEL T., et TAPSOBA D., 2002 : Rainfall variability in West Africa during the years 1950-90. J. Climate, 15, 187-202.

LEDUC C., FAVREAU G., SHROETER P., 2001 : Long term rise in a Sahelian water-table: the continental terminal in South-West Niger. J. Hydrol. 243, 43-54.

MAHE G. et OLIVRY J.-C. 1999 : Assessment of freshwater yields to the ocean along the intertropical Atlantic coast of Africa. Comptes Rendus de l'Académie des Sciences, Series Iia, $328,621-626$. 
MAHE G., LEDUC C., AMANI A., PATUREL J.-E., GIRARD S., SERVAT E., DEZETTER A., 2003 : Recent increase in surface runoff in the Sudanese Sahel and its impact on water resources. In: Hydrology of the Mediterranean and Semi-Arid Regions; Servat E., Najem W. , Leduc C., Shakeel A. (Eds.); IAHS pub. 278, 215-222.

MAHE G., PATUREL J.-E., SERVAT E., CONWAY D., DEZETTER A., 2005 : Impact of land use change on soil water holding capacity and river modelling of the Nakambe River in Burkina-Faso. J. Hydrol., 300, 33-43.

MAHE G., DIELLO P., PATUREL J.-E., BARBIER B., KARAMBIRI H., DEZETTER A., DIEULIN C., ROUCHE N., 2010 : Baisse des pluies et augmentation des écoulements au Sahel : impact climatique et anthropique sur les écoulements du Nakambe au Burkina-Faso. Sécheresse, 21, 4, 330-332.

MAHE G., 2009 : Surface/groundwater interactions in the Bani and Nakambe rivers, tributaries of the Niger and Volta basins, West Africa. Hydrol. Sci. J., 54, 704-712.

MAHE G., PATUREL J.-E., 2009 : 1896-2006 Sahelian annual rainfall variability and runoff increase of Sahelian rivers. C.R. Geosciences, 341, 538-546. doi:10.1016/j.crte.2009.05.002.

MAHE G., LIENOU G., BAMBA F., PATUREL J.-E., ADEAGA O., DESCROIX L., MARIKO A., OLIVRY J.-C., SANGARE S., 2011 : Niger river and climate change over 100 years. In: Hydro-climatology: Variability and Change, S.W. Franks, E. Boegh, E. Blyth, D.M. Hannah, K.K. Yilmaz (Eds.). Proceedings of symposium J-H02 held during IUGG2011 in Melbourne, Australia, IAHS Pub. 344, 131-137.

MAMADOU I., 2012 : La dynamique des koris et l'ensablement de leur lit et de celui du fleuve Niger dans la région de Niamey. Thèse en cotutelle Univ. Paris 1 - UAM Niamey, 250 pages.

NICHOLSON S., 2005 : On the question of the "recovery" of the rains in the West African Sahel. Journal of Arid Environments, 63, 615-641.

OLIVRY J.-C., 2002 : Synthèse des connaissances hydrologiques et potentiel en ressources en eau du fleuve Niger. World Bank, Niger Basin Authority, provisional report, 160 p, Niamey.

PANTHOU G., 2013 : Analyse des extrêmes pluviométriques en Afrique de l'Ouest et de leur évolution au cours des 60 dernières années. Thèse de doctorat, UJF - Grenoble 1, 230 pages.

PANTHOU G., VISCHEL T., et LEBEL T. 2013 : From pointwise testing to a regional vision: an integrated statistical approach to detect non stationarity in extreme daily rainfall. Application to the Sahelian region. Journal of Geophysical Research, in press.

SIGHOMNOU D., DESCROIX L., GENTHON P., MAHE G., BOUZOU MOUSSA I., GAUTIER E., MAMADOU I., VANDERVAERE J.-P., BACHIR T., COULIBALY B., RAJOT J.-L., MALAM ISSA O., MALAM ABDOU M., DESSAY N., DELAITRE E., FARAN MAIGA O., DIEDHIOU A., PANTHOU G., VISCHEL T., YACOUBA H., KARAMBIRI H., PATUREL J.-E., DIELLO P., MOUGIN E., KERGOAT L., HIERNAUX P., 2013 : La crue de 2012 à Niamey : un paroxysme du paradoxe du Sahel ? Sécheresse, 24(1), 1-11. doi : 10.1684/sec.2013.0370.

TSCHAKERT P., SAGOE R., OFORI-DARKO G. \& CODJOE S.M., 2010 : Floods in the Sahel: an analysis of anomalies, memory, and participatory learning. Climatic Change, 103, 471-502. doi: 10.1007/s10584-009-9776-y.

VAN DE WATT H.V.H. et VALENTIN C., 1992 : Soil crusting: the African view. In: M.E. Summer and B.A. Stewart (Editors), Soil Crusting, Chemical and Physical Processes, Advances in Soil Science. Lewin Publ., Boca Raton, USA, 301-338.

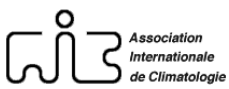

